\title{
The study of the solution of a Fredholm-Volterra integral equation by Picard operators
}

\author{
Maria Dobriţoiu
}

\begin{abstract}
In this paper we will use the Picard operators technique, in order to establish the existence and uniqueness, data dependence and Gronwall-type results for the solutions of a Fredholm-Volterra functional-integral equation. The paper ends with a result of the Ulam-Hyers stability of this integral equation.
\end{abstract}

Mathematics Subject Classification (2010): 45G10, 47H10.

Keywords: Picard operators, Fredholm integral equation, Volterra integral equation, data dependence, integral inequalities, Ulam-Hyers stability.

\section{Introduction}

The theory of integral equations has many applications in describing of numerous phenomena and problems from different research fields of the surrounding world, such as: mathematical physics, engineering, biology, economics and others. In what follows, we consider the following Fredholm-Volterra functional-integral equation:

$$
x(t)=F\left(t, g(t, x(t)), I_{F r}\left(t, s, a, b, x, K_{1}, h_{1}\right), I_{V o}\left(t, s, a, x, K_{2}, h_{2}\right)\right),
$$

where we denote:

$$
\begin{aligned}
I_{F r}\left(t, s, a, b, x, K_{1}, h_{1}\right) & =\int_{a}^{b} K_{1}(t, s) \cdot h_{1}(s, x(s), x(a), x(b)) d s \\
I_{V o}\left(t, s, a, x, K_{2}, h_{2}\right) & =\int_{a}^{t} K_{2}(t, s) \cdot h_{2}(s, x(s), x(a)) d s
\end{aligned}
$$

and

$$
\begin{aligned}
& F:[a, b] \times \mathbb{R}^{3} \rightarrow \mathbb{R}, K_{1}, K_{2}:[a, b] \times[a, b] \rightarrow \mathbb{R}, \\
& h_{1}:[a, b] \times \mathbb{R}^{3} \rightarrow \mathbb{R}, h_{2}:[a, b] \times \mathbb{R}^{2} \rightarrow \mathbb{R}, g:[a, b] \times \mathbb{R} \rightarrow \mathbb{R},
\end{aligned}
$$

and we will apply the Picard operators technique to prove the existence and uniqueness, data dependence, comparison and Gronwall-type results for the solution of the 
equation (1.1). Many authors have applied this technique to study the functionalintegral equations of mixed type (see [1], [2], [6], [9], [19], [27], etc.). Also, many authors studied the functional-integral equations of Fredholm and Volterra type and we mention some of them (see [1], [3], [7], [8], [10], [11], [12], [13], [14], [16], [17], [18] [23], [24], [25], [26], [28], etc.).

In this paper we will use the notations from [22], [23] and [25] and we recall some of them.

Let $(X, d)$ be a metric space and $A: X \rightarrow X$ an operator. We have:

$P(X):=\{Y \subset X / Y \neq \emptyset\}-$ the set of all nonempty subsets of $X$,

$I(A):=\{Y \in P(X) / A(Y) \subset Y\}$ - the family of the nonempty subsets of $X$, invariant for $A$,

$F_{A}:=\{x \in X \mid A(x)=x\}-$ the fixed points set of $A$.

Also, we denote by $A^{0}:=1_{X}, A^{1}:=A, A^{n+1}:=A \circ A^{n}, n \in N$ - the iterate operators of $A$.

Below, we present the definitions of Picard operator, c-Picard operator and weakly Picard operator.

Definition 1.1. Let $(X, d)$ be a metric space. An operator $A: X \rightarrow X$ is called Picard operator (briefly PO) if there exists $x^{*} \in X$ such that:

(a) $F_{A}=\left\{x^{*}\right\}$;

(b) the sequence $\left(A^{n}\left(x_{0}\right)\right)_{n \in \mathbb{N}}$ converges to $x^{*}$, for all $x_{0} \in X$.

Definition 1.2. Let $(X, d)$ be a metric space and $c>0$. An operator $A: X \rightarrow X$ is called c-Picard operator (briefly c-PO) if $A$ is $\mathrm{PO}$ and

$$
d\left(x, x^{*}\right) \leq c \cdot d(x, A(x)) \text { for all } x \in X .
$$

Definition 1.3. Let $(X, d)$ be a metric space. An operator $A: X \rightarrow X$ is called weakly Picard operator (briefly WPO) if the sequence $\left(A^{n}\left(x_{0}\right)\right)_{n \in \mathbb{N}}$ converges for all $x_{0} \in X$ and the limit (which may depend on $x_{0}$ ) is a fixed point of $\mathrm{A}$.

If $A$ is a WPO, then it can be considered the operator $A^{\infty}: X \rightarrow X$, defined by

$$
A^{\infty}(x):=\lim _{n \rightarrow \infty} A^{n}(x)
$$

and we observe that $A^{\infty}(X)=F_{A}$.

In addition, if $A$ is a $\mathrm{PO}$ and we denote by $x^{*}$ its unique fixed point, then $A^{\infty}(x)=x^{*}$, for all $x \in X$.

In the second section we study the existence and uniqueness of the solution of the integral equation (1.1).

In order to obtain the presented results of this section, we applied the Picard operators technique and the Contraction Principle.

Theorem 1.4 (Contraction Principle). Let $(X, d)$ be a complete metric space and $A$ : $X \rightarrow X$ an $\alpha$-contraction $(\alpha<1)$. Under these conditions we have:

(i) $F_{A}=\left\{x^{*}\right\}$

(ii) $x^{*}=\lim _{n \rightarrow \infty} A^{n}\left(x_{0}\right)$, for all $x_{0} \in X$; 
(iii) $d\left(x^{*}, A^{n}\left(x_{0}\right)\right) \leq \frac{\alpha^{n}}{1-\alpha} d\left(x_{0}, A\left(x_{0}\right)\right)$.

In order to obtain several Gronwall-type and comparison results for the solution of the integral equation (1.1), in the third section we will use the Abstract Comparison Lemma, the Abstract Gronwall Lemma and the Abstract Gronwall-Comparison Lemma, which we present below.

Lemma 1.5. (see [25]) Let $(X, d, \leq)$ be an ordered metric space and $A: X \rightarrow X$ an operator. If:

(i) $A$ is an increasing operator;

(ii) the operator $A$ is a WPO,

then the operator $A^{\infty}$ is increasing.

Lemma 1.6 (Abstract Comparison Lemma). (see [22], [23], [25]) Let A, B, C:X $\rightarrow X$ be three operators defined on the ordered metric space $(X, d, \leq)$. If:

(i) $A \leq B \leq C$;

(ii) $A, B, C$ are WPOs;

(iii) the operator $B$ is increasing,

then

$$
x \leq y \leq z \Rightarrow A^{\infty}(x) \leq B^{\infty}(y) \leq C^{\infty}(z) .
$$

Remark 1.7. Let $A, B, C$ be the operators defined in the Abstract Comparison Lemma. In addition, we suppose that B is PO, i.e. $F_{B}=\left\{x_{B}^{*}\right\}$. Then we have

$$
A^{\infty}(x) \leq x_{B}^{*} \leq C^{\infty}(x), \text { for all } x \in X .
$$

But $A^{\infty}(X)=F_{A}$ and $C^{\infty}(X)=F_{C}$ and therefore $F_{A} \leq x_{B}^{*} \leq F_{C}$.

Lemma 1.8 (Abstract Gronwall Lemma). (see [22], [23], [25]) Let $A: X \rightarrow X$ be an operator defined on the ordered metric space $(X, d, \leq)$. If:

( $i)$ the operator $A$ is $P O$ and denote by $x_{A}^{*}$ the unique fixed point of $A$;

(ii) $A$ is an increasing operator,

then

(a) $x \leq A(x) \Rightarrow x \leq x_{A}^{*}$;

(b) $x \geq A(x) \Rightarrow x \geq x_{A}^{*}$.

Lemma 1.9 (Abstract Gronwall-Comparison Lemma). (see [22], [23], [25]) Let $A_{1}, A_{2}$ : $X \rightarrow X$ be two operators defined on the ordered metric space $(X, d, \leq)$. We assume that:

(i) $A_{1}$ is increasing;

(ii) $A_{1}$ and $A_{2}$ are POs;

(iii) $A_{1} \leq A_{2}$.

If we denote by $x_{2}^{*}$ the unique fixed point of $A_{2}$, then

$$
x \leq A_{1}(x) \Rightarrow x \leq x_{2}^{*} .
$$

In the section 4 we prove a result of the continuous data dependence of the solution of the integral equation (1.1) using the General Data Dependence Theorem. 
Theorem 1.10 (General Data Dependence Theorem). Let $(X, d)$ be a complete metric space, $A, B: X \rightarrow X$ two operators and suppose:

(i) $A$ is c-PO with respect to the metric $d$ and $F_{A}=\left\{x_{A}^{*}\right\}$;

(ii) there exists $x_{B}^{*} \in F_{B}$;

(iii) there exists $\eta>0$ such that $d(A(x), B(x)) \leq \eta$, for all $x \in X$.

Under these conditions we have:

$$
d\left(x_{A}^{*}, x_{B}^{*}\right) \leq c \cdot \eta .
$$

The last section of this paper contains a result concerning the Ulam-Hyers stability of the integral equation (1.1).

Definition 1.11. (I.A. Rus [21]) Let $(X, d)$ be a metric space and $A: X \rightarrow X$ an operator. The equation of fixed point

$$
x=A(x) .
$$

is Ulam-Hyers stable if there exists a real number $c_{A}>0$ such that for each $\varepsilon>0$ and each solution $y^{*}$ of the inequation

$$
d(y, A(y)) \leq \varepsilon,
$$

there exists a solution $x^{*}$ of equation (1.2) such that

$$
d\left(y^{*}, x^{*}\right) \leq c_{A} \cdot \varepsilon .
$$

Also, in this section we will use the Remark 2.1 from I.A. Rus [21], that you can find below.

Remark 1.12. (I.A. Rus [21], Remark 2.1) If $A$ is a c-weakly Picard operator, then the fixed point equation (1.2) is Ulam-Hyers stable.

Indeed, let $\varepsilon>0$ and $y^{*}$ a solution of $d(y, A(y)) \leq \varepsilon$. Since $A$ is c-weakly Picard operator, we have that

$$
d\left(x, A^{\infty}(x)\right) \leq c \cdot d(x, A(x)), \text { for all } x \in X .
$$

If we take $x:=y^{*}$ and $x^{*}:=A^{\infty}(y)$, then we have that $d\left(y^{*}, x^{*}\right) \leq c_{A} \cdot \varepsilon$ (see [20], $[21])$.

\section{Existence and uniqueness}

In this section we present several results of existence and uniqueness for the solution of the integral equation (1.1). These results were obtained by applying the known standard techniques as in [1], [2], [5], [6] for particular integral equations.

We suppose that the following conditions are fulfilled:

$\left(a_{1}\right) K_{1}, K_{2} \in C([a, b] \times[a, b]), h_{1} \in C\left([a, b] \times \mathbb{R}^{3}\right), h_{2} \in C\left([a, b] \times \mathbb{R}^{2}\right), g \in C([a, b] \times \mathbb{R}) ;$ $\left(a_{2}\right) \quad F \in C\left([a, b] \times \mathbb{R}^{3}\right)$.

Theorem 2.1. We assume that the conditions $\left(a_{1}\right)$ and $\left(a_{2}\right)$ are satisfied. In addition we assume that: 
(i) there exist $\alpha, \beta, \gamma>0$, such that:

$\left|F\left(t, u_{1}, v_{1}, w_{1}\right)-F\left(t, u_{2}, v_{2}, w_{2}\right)\right| \leq \alpha\left|u_{1}-u_{2}\right|+\beta\left|v_{1}-v_{2}\right|+\gamma\left|w_{1}-w_{2}\right|$,

for all $t \in[a, b], u_{i}, v_{i}, w_{i} \in \mathbb{R}, i=1,2$;

(ii) there exist $L_{1}, L_{2}, L_{3}>0$ such that:

$\left|h_{1}\left(s, u_{1}, u_{2}, u_{3}\right)-h_{1}\left(s, v_{1}, v_{2}, v_{3}\right)\right| \leq L_{1}\left(\left|u_{1}-v_{1}\right|+\left|u_{2}-v_{2}\right|+\left|u_{3}-v_{3}\right|\right)$,

for all $s \in[a, b], u_{i}, v_{i} \in \mathbb{R}, i=1,2,3$;

$$
\left|h_{2}\left(s, u_{1}, u_{2}\right)-h_{2}\left(s, v_{1}, v_{2}\right)\right| \leq L_{2}\left(\left|u_{1}-v_{1}\right|+\left|u_{2}-v_{2}\right|\right),
$$

for all $s \in[a, b], u_{i}, v_{i} \in \mathbb{R}, i=1,2$;

$$
\left.|g(t, u)-g(t, v)| \leq L_{3}|u-v|\right)
$$

for all $t \in[a, b], u, v \in \mathbb{R}$

(iii) $\alpha L_{3}+\left(3 \beta M_{1} L_{1}+2 \gamma M_{2} L_{2}\right)(b-a)<1$,

where we denoted by $M_{1}$ and $M_{2}$ respectively, two positive constants, such that $\left|K_{1}(t, s)\right| \leq M_{1}$ and $\left|K_{2}(t, s)\right| \leq M_{2}$, for all $t, s \in[a, b]$.

Under these conditions the integral equation (1.1) has a unique solution $x^{*} \in C[a, b]$, that can be obtained by the successive approximations method starting at any element $x_{0} \in C[a, b]$.

In addition, if $x_{n}$ is the $n$-th successive approximation, then we have:

$$
\left\|x^{*}-x_{n}\right\|_{C} \leq \frac{\left[\alpha L_{3}+\left(3 \beta M_{1} L_{1}+2 \gamma M_{2} L_{2}\right)(b-a)\right]^{n}}{1-\alpha L_{3}-\left(3 \beta M_{1} L_{1}+2 \gamma M_{2} L_{2}\right)(b-a)} \cdot\left\|x_{0}-x_{1}\right\|_{C} .
$$

Proof. Let $X=\left(C[a, b],\|\cdot\|_{C}\right)$ be a Banach space, where $\|\cdot\|_{C}$ is the Chebyshev's norm

$$
\|x\|_{C}=\max _{t \in[a, b]}|x(t)|, \text { for all } x \in C[a, b] .
$$

Also, we consider the operator $A: X \rightarrow X$, defined by the relation:

$$
A(x)(t)=F\left(t, g(t, x(t)), I_{F r}\left(t, s, a, b, x, K_{1}, h_{1}\right), I_{V o}\left(t, s, a, x, K_{2}, h_{2}\right)\right)
$$

for all $t \in[a, b]$.

The set of the solutions of the integral equation (1.1) coincides with the set of fixed points of the operator A. From Contraction Principle it results that the operator $A$ must be a contraction. We have:

$$
\begin{aligned}
|A(x)(t)-A(y)(t)| & =\mid F\left(t, g(t, x(t)), I_{F r}\left(t, s, a, b, x, K_{1}, h_{1}\right), I_{V o}\left(t, s, a, x, K_{2}, h_{2}\right)\right) \\
& -F\left(t, g(t, y(t)), I_{F r}\left(t, s, a, b, y, K_{1}, h_{1}\right), I_{V o}\left(t, s, a, y, K_{2}, h_{2}\right)\right) \mid .
\end{aligned}
$$

From (i) and (ii) and using the Chebyshev's norm it results

$$
\|A(x)-A(y)\|_{C[a, b]} \leq\left[\alpha L_{3}+\left(3 \beta M_{1} L_{1}+2 \gamma M_{2} L_{2}\right)(b-a)\right]\|x-y\|_{C[a, b]}
$$

Consequently, from (iii) it results that the operator $A$ is an $L_{A}$-contraction with the coefficient

$$
L_{A}=\alpha L_{3}+\left(3 \beta M_{1} L_{1}+2 \gamma M_{2} L_{2}\right)(b-a) .
$$

Now, from Contraction Principle it results that the operator $A$ has a unique fixed point $F_{A}=\left\{x^{*}\right\}$ and consequently, the integral equation (1.1) has a unique solution $x^{*} \in C[a, b]$; this solution can be obtained by the successive approximations method 
starting at any element $x_{0} \in C[a, b]$ and, if $x_{n}$ is the n-th successive approximation, then the estimation (2.1) is true. The proof is complete.

Remark 2.2. In order to obtain the Theorem 2.1, of existence and uniqueness of the solution of the integral equation (1.1) in the space $C[a, b]$, we reduced the problem of determination of the solutions of this integral equation to a fixed point problem. Under the conditions of the Theorem 2.1, the operator A, defined by (2.2), is PO.

Remark 2.3. If we consider the Banach space $X=\left(C[a, b],\|\cdot\|_{B}\right)$, where $\|\cdot\|_{B}$ is the Bielecki's norm:

$$
\|x\|_{B}=\max _{t \in[a, b]}|x(t)| e^{-\tau(t-a)}
$$

for all $x \in C[a, b]$, and $\tau>0$ a parameter, and the operator $A: X \rightarrow X$, defined by (2.2), then we have another theorem of existence and uniqueness of the solution of the integral equation (1.1) in the space $C[a, b]$, that we present below.

Theorem 2.4. We assume that the conditions $\left(a_{1}\right)$ and $\left(a_{2}\right)$ are satisfied and also, the conditions (i) and (ii) from Theorem 2.1 are fulfilled. Under these conditions the integral equation (1.1) has a unique solution $x^{*} \in C[a, b]$.

Proof. We have

$$
\begin{aligned}
|A(x)(t)-A(y)(t)| & \leq \alpha L_{3} e^{\tau(t-a)}\|x-y\|_{B}+3 \frac{\beta M_{1} L_{1}}{\tau} e^{\tau(t-a)}\|x-y\|_{B} \\
& +2 \frac{\gamma M_{2} L_{2}}{\tau} e^{\tau(t-a+b-t)}\|x-y\|_{B}
\end{aligned}
$$

and therefore, using the Bielecki's norm, we obtain:

$$
\|A(x)-A(y)\|_{B} \leq\left[\alpha L_{3}+3 \frac{\beta M_{1} L_{1}}{\tau}+2 \frac{\gamma M_{2} L_{2}}{\tau} e^{\tau(b-a)}\right]\|x-y\|_{B} .
$$

It is clear that one can find a positive parameter $\tau$, such that

$$
\alpha L_{3}+3 \frac{\beta M_{1} L_{1}}{\tau}+2 \frac{\gamma M_{2} L_{2}}{\tau} e^{\tau(b-a)}<1,
$$

and thus $A$ is an $L_{A}$-contraction with

$$
L_{A}=\alpha L_{3}+3 \frac{\beta M_{1} L_{1}}{\tau}+2 \frac{\gamma M_{2} L_{2}}{\tau} e^{\tau(b-a)}
$$

and the conclusion of theorem is obtained by applying the Contraction Principle (Theorem 1.4).

Example 2.5. The following equation is a particular case of the integral equation (1.1), when $g(t, x(t))=x(t)$ :

$$
x(t)=F\left(t, x(t), I_{F r}\left(t, s, a, b, x, K_{1}, h_{1}\right), I_{V o}\left(t, s, a, x, K_{2}, h_{2}\right)\right),
$$

where we used the same notations for $I_{F r}$ and $I_{V o}$ as at the beginning of the first section.

Let us consider this integral equation in the following hypotheses:

(i) $F \in C\left([a, b] \times \mathbb{R}^{3}\right), K_{1}, K_{2} \in C([a, b] \times[a, b]), h_{1} \in C\left([a, b] \times \mathbb{R}^{3}\right)$,

$h_{2} \in C\left([a, b] \times \mathbb{R}^{2}\right)$; 
(ii) there exist $\alpha, \beta, \gamma>0$, such that:

$$
\left|F\left(t, u_{1}, v_{1}, w_{1}\right)-F\left(t, u_{2}, v_{2}, w_{2}\right)\right| \leq \alpha\left|u_{1}-u_{2}\right|+\beta\left|v_{1}-v_{2}\right|+\gamma\left|w_{1}-w_{2}\right|,
$$

for all $t \in[a, b], u_{i}, v_{i}, w_{i} \in \mathbb{R}, i=1,2$;

(iii) there exist $L_{1}, L_{2}>0$, such that:

$$
\left|h_{1}\left(s, u_{1}, u_{2}, u_{3}\right)-h_{1}\left(s, v_{1}, v_{2}, v_{3}\right)\right| \leq L_{1}\left(\left|u_{1}-v_{1}\right|+\left|u_{2}-v_{2}\right|+\left|u_{3}-v_{3}\right|\right),
$$

for all $s \in[a, b], u_{i}, v_{i} \in \mathbb{R}, i=1,2,3$;

$$
\left|h_{2}\left(s, u_{1}, u_{2}\right)-h_{1}\left(s, v_{1}, v_{2}\right)\right| \leq L_{2}\left(\left|u_{1}-v_{1}\right|+\left|u_{2}-v_{2}\right|\right),
$$

for all $s \in[a, b], u_{i}, v_{i} \in \mathbb{R}, i=1,2 ;$

(iv) $\alpha+\left(3 \beta M_{1} L_{1}+2 \gamma M_{2} L_{2}\right)(b-a)<1$,

where we denoted by $M_{1}$ and $M_{2}$ respectively, two positive constants, such that $\left|K_{1}(t, s)\right| \leq M_{1}$ and $\left|K_{2}(t, s)\right| \leq M_{2}$, for all $t, s \in[a, b]$.

Then the integral equation (1.1) has a unique solution $x^{*} \in C[a, b]$, that can be obtained by the successive approximations method starting at any element $x_{0} \in$ $C[a, b]$. Moreover, if $x_{n}$ is the $n$-th successive approximation, then we have:

$$
\left\|x^{*}-x_{n}\right\|_{C} \leq \frac{\left[\alpha+\left(3 \beta M_{1} L_{1}+2 \gamma M_{2} L_{2}\right)(b-a)\right]^{n}}{1-\alpha-\left(3 \beta M_{1} L_{1}+2 \gamma M_{2} L_{2}\right)(b-a)} \cdot\left\|x_{0}-x_{1}\right\|_{C} .
$$

In order to prove this result, we applied the Theorem 2.1 in particular case of

$$
g(t, x(t))=x(t)
$$

Remark 2.6. A similar result can be obtained for the solution of integral equation

$$
x(t)=F\left(t, x(a), I_{F r}\left(t, s, a, b, x, K_{1}, h_{1}\right), I_{V o}\left(t, s, a, x, K_{2}, h_{2}\right)\right),
$$

by applying the Theorem 2.1 in particular case of $g(t, x(t))=x(a)$.

Remark 2.7. In the paper [9] has been studied the existence and uniqueness of the solution of nonlinear Fredholm-Volterra functional-integral equation:

$$
x(t)=F\left(t, x(a), \int_{a}^{b} K_{1}\left(t, s, x\left(g_{1}(s)\right)\right) d s, \int_{a}^{t} K_{2}\left(t, s, x\left(g_{2}(s)\right)\right) d s\right) .
$$

\section{Comparison results and Gronwall lemmas}

We present below a comparison result and two Gronwall-type lemmas for the solution of the integral equation (1.1). These results have been obtained by using the Picard operators technique and applying the Abstract Comparison Lemma, the Abstract Gronwall Lemma and the Abstract Gronwall-Comparison Lemma as in [4], [5], [15] for particular operatorial equations.

In order to obtain a comparison result, we consider the integral equations:

$$
x(t)=F_{i}\left(t, g(t, x(t)), I_{F r}^{i}\left(t, s, a, b, x, K_{1}, h_{1}^{i}\right), I_{V o}^{i}\left(t, s, a, x, K_{2}, h_{2}^{i}\right)\right),
$$


where we denoted:

$$
\begin{aligned}
I_{F r}^{i}\left(t, s, a, b, x, K_{1}, h_{1}^{i}\right) & =\int_{a}^{b} K_{1}(t, s) \cdot h_{1}^{i}(s, x(s), x(a), x(b)) d s \\
I_{V o}^{i}\left(t, s, a, x, K_{2}, h_{2}^{i}\right) & =\int_{a}^{t} K_{2}(t, s) \cdot h_{2}^{i}(s, x(s), x(a)) d s
\end{aligned}
$$

where

$$
\begin{aligned}
& F_{i} \in C\left([a, b] \times \mathbb{R}^{3}\right), g \in C([a, b] \times \mathbb{R}), \\
& K_{1}, K_{2} \in C\left([a, b] \times[a, b], \mathbb{R}_{+}\right), h_{1}^{i} \in C\left([a, b] \times \mathbb{R}^{3}\right), \\
& h_{2}^{i} \in C\left([a, b] \times \mathbb{R}^{2}\right), i=1,2,3 .
\end{aligned}
$$

We have:

Theorem 3.1. Suppose that:

( $i$ ) the functions $F_{i}, g, K_{1}, K_{2}, h_{1}^{i}, h_{2}^{i}, i=1,2,3$ satisfy the conditions of Theorem 2.1, and let $x_{i}^{*}$ be the unique solution of the integral equation (3.1) corresponding to $F_{i}, h_{1}^{i}, h_{2}^{i}, i=1,2,3$;

(ii) the functions $F_{2}(t, \cdot, \cdot, \cdot), h_{1}^{2}(t, \cdot, \cdot, \cdot), h_{2}^{2}(t, \cdot, \cdot)$ are increasing;

(iii) $F_{1} \leq F_{2} \leq F_{3}, h_{1}^{1} \leq h_{1}^{2} \leq h_{1}^{3}$ and $h_{2}^{1} \leq h_{2}^{2} \leq h_{2}^{3}$.

Then

$$
x_{1}^{*} \leq x_{2}^{*} \leq x_{3}^{*} .
$$

Proof. We consider the Banach space $X=\left(C[a, b],\|\cdot\|_{C}\right)$ and the operators $A_{i}$ : $X \rightarrow X$, defined by the relation (2.2) corresponding to functions $F_{i}, g, K_{1}, K_{2}, h_{1}^{i}, h_{2}^{i}$, $i=1,2,3$ :

$$
A_{i}(x)(t)=F_{i}\left(t, g(t, x(t)), I_{F r}^{i}\left(t, s, a, b, x, K_{1}, h_{1}^{i}\right), I_{V o}^{i}\left(t, s, a, x, K_{2}, h_{2}^{i}\right)\right) .
$$

From condition (i) it results that the operators $A_{i}: X \rightarrow X, i=1,2,3$ are PO's and therefore each of these operators has a unique fixed point, $F_{A_{i}}=\left\{x_{i}^{*}\right\}$.

From condition (ii) we deduce that the operator $A_{2}$ is increasing and from condition (iii) we obtain that $A_{1} \leq A_{2} \leq A_{3}$.

Now, applying the Abstract Comparison Lemma (Lemma 1.6), it results that

$$
x_{1} \leq x_{2} \leq x_{3} \Longrightarrow A_{1}^{\infty}\left(x_{1}\right) \leq A_{2}^{\infty}\left(x_{2}\right) \leq A_{3}^{\infty}\left(x_{3}\right),
$$

but $A_{1}, A_{2}, A_{3}$ are PO's and then by Remark 1.7, the conclusion of this theorem follows, i.e. $x_{1}^{*} \leq x_{2}^{*} \leq x_{3}^{*}$. The proof is complete.

For the solution of the integral equation (1.1) we present below, the following two Gronwall-type lemmas.

Theorem 3.2. We suppose that:

(i) $F \in C\left([a, b] \times \mathbb{R}^{3}\right), K_{1}, K_{2} \in C\left([a, b] \times[a, b], \mathbb{R}_{+}\right), h_{1} \in C\left([a, b] \times \mathbb{R}^{3}\right)$, $h_{2} \in C\left([a, b] \times \mathbb{R}^{2}\right), g \in C([a, b] \times \mathbb{R}) ;$

(ii) $F, K_{1}, K_{2}, h_{1}, h_{2}, g$ satisfy the conditions (i)-(iii) of Theorem 2.1, and denote by $x^{*} \in C[a, b]$ the unique solution of the integral equation (1.1);

$\left(\right.$ iii) $h_{1}(s, \cdot, \cdot, \cdot): \mathbb{R}^{3} \rightarrow \mathbb{R}, h_{2}(s, \cdot, \cdot): \mathbb{R}^{2} \rightarrow \mathbb{R}$ are increasing functions for all $s \in[a, b]$; 
(iv) $F(t, \cdot, \cdot, \cdot): \mathbb{R}^{3} \rightarrow \mathbb{R}$ is increasing function for all $t \in[a, b]$.

Under these conditions, the following statements are true:

(a) if $x$ is a lower-solution of integral equation (1.1) then $x \leq x^{*}$;

(b) if $x$ is a upper-solution of integral equation (1.1) then $x \geq x^{*}$.

Proof. We consider the operator $A: X \rightarrow X$, defined by (2.2). From conditions (i) and (ii) it results that this operator is $\mathrm{PO}$ and denote by $x^{*}$ the unique fixed point of A. From the assumptions (i), (iii) and (iv) it results that the operator $A$ is increasing.

Now, the conditions of the Abstract Gronwall Lemma (Lemma 1.8), being satisfied, it results that the conclusions of this theorem:

- if $x$ is a lower-solution of the integral equation (1.1), i.e. $x \leq A(x)$, then $x \leq x^{*}$;

- if $x$ is a upper-solution of the integral equation (1.1), i.e. $x \geq A(x)$, then $x \geq x^{*}$, are true. The proof is complete.

To obtain an effective Gronwall-type lemma, it can use the Abstract GronwallComparison Lemma (Lemma 1.9), and we obtain a result that we present below.

Theorem 3.3. We consider the integral equation (1.1) corresponding to $F_{i}, g, K_{1}, K_{2}$, $h_{1}^{i}, h_{2}^{i}$, for $i=1,2$. We assume that:

(i) $F_{i} \in C\left([a, b] \times \mathbb{R}^{3}\right), K_{1}, K_{2} \in C\left([a, b] \times[a, b], \mathbb{R}_{+}\right), h_{1}^{i} \in C\left([a, b] \times \mathbb{R}^{3}\right)$,

$h_{2}^{i} \in C\left([a, b] \times \mathbb{R}^{2}\right), g \in C([a, b] \times \mathbb{R}), i=1,2 ;$

(ii) $F_{i}, g, K_{1}, K_{2}, h_{1}^{i}, h_{2}^{i}$ satisfy the conditions (i)-(iii) of Theorem 2.1, for $i=1,2$;

(iii) $h_{1}^{1}(s, \cdot, \cdot, \cdot): \mathbb{R}^{3} \rightarrow \mathbb{R}, h_{2}^{1}(s, \cdot, \cdot): \mathbb{R}^{2} \rightarrow \mathbb{R}$ are increasing functions for all $s \in[a, b]$;

(iv) $F_{1}(t, \cdot, \cdot, \cdot): \mathbb{R}^{3} \rightarrow \mathbb{R}, g(t, \cdot): \mathbb{R} \rightarrow \mathbb{R}$ are increasing functions for all $t \in[a, b]$.

(v) $F_{1} \leq F_{2}, h_{1}^{1} \leq h_{1}^{2}$ and $h_{2}^{1} \leq h_{2}^{2}$.

If $x$ is a solution of integral inequality

$$
x(t) \leq F_{1}\left(t, g(t, x(t)), I_{F r}^{1}\left(t, s, a, b, x, K_{1}, h_{1}^{1}\right), I_{V o}^{1}\left(t, s, a, x, K_{2}, h_{2}^{1}\right)\right),
$$

where

$$
\begin{aligned}
I_{F r}^{1}\left(t, s, a, b, x, K_{1}, h_{1}^{1}\right) & =\int_{a}^{b} K_{1}(t, s) \cdot h_{1}^{1}(s, x(s), x(a), x(b)) d s \\
I_{V o}^{1}\left(t, s, a, x, K_{2}, h_{2}^{1}\right) & =\int_{a}^{t} K_{2}(t, s) \cdot h_{2}^{1}(s, x(s), x(a)) d s,
\end{aligned}
$$

then $x \leq x_{2}^{*}$, where $x_{2}^{*}$ is the unique solution of integral equation (1.1) corresponding to $F_{2}, g, K_{1}, K_{2}, h_{1}^{2}, h_{2}^{2}$ :

$$
x(t)=F_{2}\left(t, g(t, x(t)), I_{F r}^{2}\left(t, s, a, b, x, K_{1}, h_{1}^{2}\right), I_{V o}^{2}\left(t, s, a, x, K_{2}, h_{2}^{2}\right)\right),
$$

where

$$
\begin{aligned}
I_{F r}^{2}\left(t, s, a, b, x, K_{1}, h_{1}^{2}\right) & =\int_{a}^{b} K_{1}(t, s) \cdot h_{1}^{2}(s, x(s), x(a), x(b)) d s \\
I_{V o}^{2}\left(t, s, a, x, K_{2}, h_{2}^{2}\right) & =\int_{a}^{t} K_{2}(t, s) \cdot h_{2}^{2}(s, x(s), x(a)) d s .
\end{aligned}
$$


Proof. We consider the operator $A_{1}, A_{2}$ defined by (2.2), corresponding to $F_{1}, g, K_{1}$, $K_{2}, h_{1}^{1}, h_{2}^{1}$ and $F_{2}, g, K_{1}, K_{2}, h_{1}^{2}, h_{2}^{2}$.

From Theorem 2.1 we have that $A_{1}$ and $A_{2}$ are POs, and we denote by $x_{i}^{*}$ the unique fixed point of operator $A_{i}, i=1,2$.

From condition (ii) it results that $A_{1}$ is increasing and from condition (iii) we obtain that $A_{1} \leq A_{2}$.

If $x$ is a solution of (3.2), then $x \leq A_{1}(x)$.

Now, we apply the Abstract Gronwall-Comparison Lemma (Lemma 1.9), and we obtain the conclusion of the theorem. The proof is complete.

\section{Data dependence}

In order to study the data dependence of the solution of the integral equation (1.1) we consider the following perturbed integral equation:

$$
x(t)=F\left(t, g(t, x(t)), I_{F r}\left(t, s, a, b, x, K_{1}, k_{1}\right), I_{V o}\left(t, s, a, x, K_{2}, k_{2}\right)\right),
$$

where

$$
\begin{aligned}
I_{F r}\left(t, s, a, b, x, K_{1}, k_{1}\right) & =\int_{a}^{b} K_{1}(t, s) \cdot k_{1}(s, x(s), x(a), x(b)) d s \\
I_{V o}\left(t, s, a, x, K_{2}, k_{2}\right) & =\int_{a}^{t} K_{2}(t, s) \cdot k_{2}(s, x(s), x(a)) d s
\end{aligned}
$$

and

$$
\begin{aligned}
& F:[a, b] \times \mathbb{R}^{3} \rightarrow \mathbb{R}, K_{1}, K_{2}:[a, b] \times[a, b] \rightarrow \mathbb{R}, \\
& k_{1}:[a, b] \times \mathbb{R}^{3} \rightarrow \mathbb{R}, k_{2}:[a, b] \times \mathbb{R}^{2} \rightarrow \mathbb{R}, g:[a, b] \times \mathbb{R} \rightarrow \mathbb{R} .
\end{aligned}
$$

We have the following data dependence theorem of the solution of the integral equation $(1.1)$ :

Theorem 4.1. Suppose that:

(i) $F, K_{1}, K_{2}, h_{1}, h_{2}, g$ satisfy the conditions of Theorem 2.1 and we denote by $x^{*} \in$ $C[a, b]$ the unique solution of integral equation (1.1);

(ii) $k_{1} \in C\left([a, b] \times \mathbb{R}^{3}\right), k_{2} \in C\left([a, b] \times \mathbb{R}^{2}\right)$;

(iii) there exists $\eta_{1}, \eta_{2}>0$ such that

$$
\begin{aligned}
& \left|h_{1}(s, u, v, w)-k_{1}(s, u, v, w)\right| \leq \eta_{1}, \text { for all } s \in[a, b], u, v, w \in \mathbb{R}, \text { and } \\
& \left|h_{2}(s, u, v)-k_{2}(s, u, v)\right| \leq \eta_{2}, \text { for all } s \in[a, b], u, v \in \mathbb{R} .
\end{aligned}
$$

Under these conditions, if $y^{*} \in C[a, b]$ is a solution of the integral equation (4.1), then we have:

$$
\left\|x^{*}-y^{*}\right\|_{C} \leq \frac{\left(M_{1} \eta_{1}+M_{2} \eta_{2}\right)(b-a)}{1-\alpha L_{3}-\left(3 \beta M_{1} L_{1}+2 \gamma M_{2} L_{2}\right)(b-a)} .
$$


Proof. We consider the operator from the proof of Theorem 2.1, $A: C[a, b] \rightarrow C[a, b]$, attached to integral equation (1.1) and defined by the relation (2.2):

$$
A(x)(t)=F\left(t, g(t, x(t)), I_{F r}\left(t, s, a, b, x, K_{1}, h_{1}\right), I_{V o}\left(t, s, a, x, K_{2}, h_{2}\right)\right),
$$

for all $t \in[a, b]$.

From condition (i) it results that the operator $A$ is a $L_{A}$-contraction with the coefficient

$$
L_{A}=\alpha L_{3}+\left(3 \beta M_{1} L_{1}+2 \gamma M_{2} L_{2}\right)(b-a)
$$

(Theorem 2.1) and therefore, $A$ is c-PO with $c=\frac{1}{1-L_{A}}$.

Also, we attach to the integral equation (4.1) the operator $B: C[a, b] \rightarrow C[a, b]$, defined by the relation:

$$
B(x)(t)=F\left(t, g(t, x(t)), I_{F r}\left(t, s, a, b, x, K_{1}, k_{1}\right), I_{V o}\left(t, s, a, x, K_{2}, k_{2}\right)\right)
$$

for all $t \in[a, b]$.

From conditions (i) and (ii) it results that the operator B is correctly defined.

The set of the solutions of the perturbed integral equation $(4.1)$ in the space $C[a, b]$ coincides with the fixed points set of the operator B defined by the relation (4.3).

We have:

$$
\begin{aligned}
|A(x)(t)-B(x)(t)| & =\mid F\left(t, g(t, x(t)), I_{F r}\left(t, s, a, b, x, K_{1}, h_{1}\right), I_{V o}\left(t, s, a, x, K_{2}, h_{2}\right)\right) \\
& -F\left(t, g(t, y(t)), I_{F r}\left(t, s, a, b, y, K_{1}, k_{1}\right), I_{V o}\left(t, s, a, y, K_{2}, k_{2}\right)\right) \mid
\end{aligned}
$$

and from condition (iii) it results that

$$
|A(x)(t)-B(x)(t)| \leq\left(M_{1} \eta_{1}+M_{2} \eta_{2}\right)(b-a), \text { for all } t \in[a, b] .
$$

Now, using the Chebyshev's norm, we obtain:

$$
\|A(x)-B(x)\|_{C} \leq\left(M_{1} \eta_{1}+M_{2} \eta_{2}\right)(b-a)
$$

and applying the General Data Dependence Theorem (Theorem 1.10), with

$$
c=\frac{1}{1-L_{A}} \text { and } \eta=\left(M_{1} \eta_{1}+M_{2} \eta_{2}\right)(b-a),
$$

it results the estimation (4.2). The proof is complete.

\section{Ulam-Hyers stability}

Theorem 5.1. Under the conditions of Theorem 2.1, the integral equation (1.1) is Ulam-Hyers stable, i.e. for $\varepsilon>0$ and $y^{*} \in C[a, b]$ a solution of the inequation

$$
\left|y(t)-F\left(t, g(t, y(t)), I_{F r}\left(t, s, a, b, y, K_{1}, h_{1}\right), I_{V o}\left(t, s, a, y, K_{2}, h_{2}\right)\right)\right| \leq \varepsilon
$$

for all $t \in[a, b]$, there exists a solution of the integral equation (1.1), $x^{*} \in C([a, b]$, such that

where

$$
\left|y^{*}(t)-x^{*}(t)\right| \leq \frac{1}{1-L_{A}} \varepsilon, \text { for all } t \in[a, b],
$$

$$
L_{A}=\alpha L_{3}+\left(3 \beta M_{1} L_{1}+2 \gamma M_{2} L_{2}\right)(b-a) .
$$


Proof. We consider the operator $A$, defined by the relation (2.2). Under the conditions of Theorem 2.1, it results that the operator $A$ is a contraction and therefore, $A$ is c-PO with the constant $c=\frac{1}{1-L_{A}}$,

$$
L_{A}=\alpha L_{3}+\left(3 \beta M_{1} L_{1}+2 \gamma M_{2} L_{2}\right)(b-a) .
$$

Now, the conclusion of this theorem is obtained as an application of the Remark 1.12 (I.A.Rus [21], Remark 2.1) anf the proof is complete.

\section{References}

[1] Andras, Sz., Ecuaţii integrale Fredholm-Volterra, Editura Didactică şi Pedagogică, Bucureşti, 2005.

[2] Calio, F., Marcchetti, E., Mureşan, V., On some Volterra-Fredholm integral equations, Int. J. Pure Appl. Math., 31(2006), no. 2, 173-184.

[3] Coman, Gh., Rus, I., Pavel, G., Rus, I. A., Introducere în teoria ecuaţiilor operatoriale, Editura Dacia, Cluj-Napoca, 1976.

[4] Crăciun, C., On some Gronwall inequalities, Seminar on Fixed Point Theory, 1(2000), 31-34.

[5] Crăciun, C., Lungu, N., Abstract and concrete Gronwall lemmas, Fixed Point Theory, 10(2009), no. 2, 221-228.

[6] Crăciun, C., Şerban, M.A., A nonlinear integral equation via Picard operators, Fixed Point Theory, 12(2011), no. 1, 57-70.

[7] Dobriţoiu, M., The solution to a Fredholm implicit integral equation in the $B(0 ; R)$ sphere, Bulletins for Applied \& Computer Mathematics, Budapest, BAM CV (2003), no. 2162, 27-32.

[8] Dobriţoiu, M., Existence and continuous dependence on data of the solution of an integral equation, Bulletins for Applied \& Computer Mathematics, Budapest, 2005.

[9] Dobriţoiu, M., A Fredholm-Volterra integral equation with modified argument, Analele Univ. din Oradea, Fasc. Matematica, tom XIII, 2006, 133-138.

[10] Dobritoiu, M., On an integral equation with modified argument, Acta Univ. Apulensis Math. Inform., 2006, no. 11, 387-391.

[11] Dobriţoiu, M., Analysis of an integral equation with modified argument, Stud. Univ. Babeş-Bolyai Math., 51(2006), no. 1, 81-94.

[12] Dobriţoiu, M., Properties of the solution of an integral equation with modified argument, Carpathian J. Math., 23(2007), no. 1-2, 70-80.

[13] Dobriţoiu, M., A nonlinear Fredholm integral equation, Transylvanian Journal of Mathematics and Mechanics, 1(2009), no. 1-2, 25-32.

[14] Dobritoiu, M., A class of nonlinear integral equations, Transylvanian Journal of Mathematics and Mechanics, 4(2012), no. 2, 117-123.

[15] Lungu, N., On some Volterra integral inequalities, Fixed Point Theory, 8(2007), no. 1, 39-45.

[16] Olaru, I.M., An integral equation via weakly Picard operators, Fixed Point Theory, 11(2010), no. 1, 97-106.

[17] Olaru, I.M., Data dependence for some integral equations, Stud. Univ. Babeş-Bolyai Math., 55(2010), no. 2, 159-165. 
[18] Olaru, I.M., On some integral equations with deviating argument, Stud. Univ. BabeşBolyai Math., 50(2005), no. 4, 65-72.

[19] Petruşel, A., Fredholm-Volterra integral equations and Maia's theorem, Seminar on Fixed Point Theory, Babeş-Bolyai University Cluj-Napoca, 1988, 79-82.

[20] Rus, I.A., Results and problems in Ulam stability of operatorial equations and inclusions. Handbook of functional equations, Springer Optim. Appl., 96(2014), 323-352.

[21] Rus, I.A., Remarks on Ulam stability of the operatorial equations, Fixed Point Theory, 10(2009), 305-320.

[22] Rus, I.A., Fixed points, upper and lower fixed points: abstract Gronwall lemmas, Carpathian J. Math., 20(2004), no. 1, 125-134.

[23] Rus, I.A., Picard operators and applications, Sci. Math. Jpn., 58(2003), no. 1, 191-219.

[24] Rus, I.A., Generalized Contractions and Applications, Cluj University Press, ClujNapoca, 2001.

[25] Rus, I.A., Weakly Picard operators and applications, Seminar on Fixed Point Theory, Babeş-Bolyai University Cluj-Napoca, 2(2001), 41-58.

[26] Rus, I.A., Principii şi Aplicaţii ale Teoriei Punctului Fix, Editura Dacia, Cluj-Napoca, 1979.

[27] Sincelean, A., On a class of functional-integral equations, Seminar on Fixed Point Theory, Babeş-Bolyai University Cluj-Napoca, 1(2000), 87-92.

[28] Şerban, M.A., Teoria Punctului Fix pentru Operatori Definiţi pe Produs Cartezian, Presa Universitară Clujeană, Cluj-Napoca, 2002.

Maria Dobriţoiu

University of Petroşani

Faculty of Sciences

20, Universităţii Street

302006 Petroşani, Romania

e-mail: mariadobritoiu@yahoo.com 